\title{
Caregiver Involvement in MS: Duty or Disruption?
}

\author{
Jürg Kesselring • Alexey Boyko (D) - Alice Laroni (i) - Trishna Bharadia (D) · \\ Pieter van Galen (1) · Nektaria Alexandri
}

Received: May 18, 2021 / Accepted: November 1, 2021 / Published online: November 18, 2021

(c) The Author(s) 2021

\begin{abstract}
Multiple sclerosis (MS) is a complex condition with numerous physical, cognitive and emotional symptoms. These may necessitate significant, permanent lifestyle changes for people with multiple sclerosis (PwMS) and their caregivers and
\end{abstract}

J. Kesselring $(\bowtie)$

Department of Neurology and Neurorehabilitation, Kliniken Valens, Valens, Switzerland

e-mail: Juerg.Kesselring@kliniken-valens.ch

A. Boyko

Institute of Clinical Neurology and Department of Neuroimmunology of the Federal Centre of Brain Research and Neurotechnologies, Pirogov's Russian National Research Scientific Medical University, Moscow, Russia

\section{A. Laroni}

Department of Neuroscience, Rehabilitation, Ophthalmology, Genetics, Maternal and Child Health, University of Genoa, Genoa, Italy

\section{A. Laroni}

IRCCS Ospedale Policlinico San Martino, Genoa, Italy

T. Bharadia

Patient Author, Member of the MS in the 21st

Century Steering Group, Buckinghamshire, UK

P. van Galen

Patient Author, Member of the MS in the 21st

Century Steering Group, Overijse, Belgium

N. Alexandri

Merck KGaA, Darmstadt, Germany families, meaning it is important in contemporary neurological practice to consider including families and/or caregivers in the management of MS. However, existing evidence suggests that family involvement is not always beneficial; for example, it can exert either a strong positive or negative influence on the ability of PwMS to achieve optimal outcomes from their treatment and disease management. This paper, based on a live debate between neurologists and PwMS, examines the current perceptions on constructive involvement of families and caregivers in consultations for and management of MS, and reveals several areas where additional studies are warranted. Shared decision-making in MS has historically been a collaboration solely between healthcare professionals (HCPs) and PwMS, but PwMS are now more frequently being accompanied to appointments by a support person. This paper encourages HCPs to understand the dynamics between PwMS and their support person, and to individualize consultations and information accordingly. Family and caregiver involvement in the provision of care for PwMS needs to be for the benefit of, and at the discretion of, the PwMS. Support for families of PwMS, although important, may be more effectively and appropriately delivered through other channels outside of the clinical setting. Educating HCPs on the current patient experience to enable them to provide improved personalized care will ensure a mutualistic, patient-centred relationship with PwMS, which 
will help to optimize outcomes. Communication tools may also facilitate these interactions.

\section{PLAIN LANGUAGE SUMMARY}

Multiple sclerosis (MS) is a highly variable condition. The uncertainty this brings can affect the mental well-being of the entire family of someone living with MS. Additionally, people with MS may need help administering and remembering to use medicines, and require changes to the way they live their life (e.g. employment decisions, home adjustments and care), which can also disrupt the family's lifestyle. The family undoubtedly needs to be involved in certain decisions, such as family planning, or when caring for very young or old people with MS. However, extensive family involvement may not always be a good thing for all people with MS. Therefore, it is the responsibility of healthcare professionals to understand the pivotal role of the caregiver and seek appropriate opportunities to engage with family and support persons on a case-by-case basis. To highlight the importance of family members in the management of MS, an international group of expert neurologists and people with MS discussed the best ways to involve families in consultations and decisionmaking, without disrupting care or undermining the independence of the people with MS. The group stresses that a family's involvement in the provision of care needs to be for the benefit of the people with MS and that many factors can influence whether family involvement is perceived positively or negatively by the people with MS. Support for the family is important; however, it may be more appropriately delivered outside the healthcare professional consultation, and instead provided by a patient or caregiver organization.

Keywords: Multiple sclerosis; Communication; Adherence; Care satisfaction; Quality of life; Relationships; Consultation style; Shared care, caregiver

\section{Key Summary Points}

\section{Why carry out the study?}

The variable and unpredictable nature of multiple sclerosis (MS) is accompanied by unique challenges for both people with MS (PwMS) and their caregivers.

PwMS-caregiver relationships are often very important when PwMS make treatment decisions, especially when a PwMS is experiencing MS-related cognitive impairment. Understanding the dynamics and potential influences these relationships have will allow healthcare professionals (HCPs) to improve strategies for managing shared decision-making in order to provide the best patient outcomes.

\section{What was learned from the study?}

Caregiver involvement during MS care and consultations can bridge gaps in communication between PwMS and HCPs, offering HCPs a more objective perspective and promoting rational decision-making. Caregivers also provide emotional support for PwMS which can help maintain patient engagement and treatment adherence.

The presence of a caregiver in the decisionmaking process can place further pressure on the HCP who must consider their potentially competing priorities.

Furthermore, it may inhibit the PwMS from being open and involved for fear of embarrassment or an over-reliance on a caregiver who may be overbearing.

HCPs must recognize there is no 'one-sizefits-all' approach to incorporating caregivers. Potential strategies include consulting patients and caregivers separately, whilst ensuring an overall consistent and sensitive approach to sharing information. 


\section{INTRODUCTION}

As a chronic condition, multiple sclerosis (MS) has an impact on not only the life of people with MS (PwMS) but also that of their families. Additionally, the intrinsically highly variable trajectory of MS makes predicting short- and long-term disease activity, disease evolution and disability progression extremely difficult [1-3]. This prognostic uncertainty can increase the challenges faced by PwMS and their relatives $[2,4]$ when adjusting to and learning to live with the condition. PwMS may need support from their family in administering and remembering to use disease-modifying therapies (DMTs) and adhering to broader treatment plans. MS is a complex condition with numerous physical, cognitive and emotional symptoms [3] that may in some cases necessitate significant changes to everyday life for PwMS (e.g. employment decisions, home adjustments and care), which in turn can disrupt the entire family's plans and lifestyle $[4,5]$.

Shared decision-making is a process where patients and healthcare professionals (HCPs) work together to determine the most appropriate treatment or other element of care with consideration to the patient's values, goals, preferences and circumstances $[6,7]$. As a result, shared decision-making increases patient autonomy, education and satisfaction, while reducing conflict about decisions and encouraging mutualistic, patient-centred care [6]. Shared decision-making has been shown to help PwMS adapt to their new way of life, optimizing day-to-day management and improving adherence to treatment [8-10]. While shared decision-making has historically been viewed as a partnership solely between PwMS and HCPs, certain situations may also require the involvement of family members. This involvement may be driven by a personal or cultural preference of the PwMS; the cultural aspects of family involvement require further research, given that although MS is relatively well studied in North America and parts of Europe, it is generally not so well studied in other regions where the prevalence and incidence of MS are increasing, including in parts of Eastern and Southern
Europe [11-13], Asia [14, 15] and Latin America $[16,17]$. In addition, more recently, a more direct decision-making need may exist in cases of pregnancy and family planning [18], which have become more important elements to consider with the large improvement in MS care and understanding of MS in pregnancy in recent years. Services for adolescent PwMS are necessary to consider as MS is increasingly recognized in this population [19].

Although development of a shared decisionmaking approach between PwMS and HCPs has been a focus of recent publications [20], disparities remain in HCPs' and PwMS' perceptions of treatment and associated challenges, as well as expectations of, and satisfaction with care [21]. Cognitive impairment is a symptom that can emerge early in the course of the disease $[22,23]$, and can worsen adherence, academic performance and social quality of life $[19,24]$. Cognitive impairment may further increase the potential disparities between PwMS and HCPs, limit the development of a shared understanding, and consequently have a negative impact on the beneficial shared decision-making process. In these situations, the active involvement of a family member may help both the PwMS and HCPs to regain their shared understanding, improving engagement and education, and facilitating informed consent to be treated with DMTs or other aspects of care. Nevertheless, to practically implement and optimize a family's involvement in the care of PwMS may not always be straightforward.

Recognizing that MS has a lifelong impact on both the PwMS and their family, discussions about the extent to which family members should be involved in consultations between HCPs and PwMS, and how best to meet the relatives' needs, are particularly important in contemporary neurological practice. This report summarizes a debate between a panel of MS in the 21st Century [25] Steering Group members and an audience of HCPs, PwMS and caregivers, with a goal of re-evaluating how family involvement can be optimized in the current and evolving MS landscape, including exploration of increasingly relevant topics, such as care of paediatric PwMS. Areas which require further study are also highlighted. The debate 
was held at the 27th Annual Meeting of the European Charcot Foundation in Baveno, Italy on November 21, 2019. The panel consisted of neurologists Professor Alexey Boyko and Dr Alice Laroni, and two PwMS, Trishna Bharadia and Pieter van Galen, and the meeting was chaired by Professor Jürg Kesselring.

The insights from the meeting, summarized in this paper, aim to challenge current perceptions of what characterizes shared decisionmaking in the provision of care for PwMS, highlight the potentially significant influence that family members can have on patient engagement and satisfaction with their care, and encourage HCPs to consider how best to integrate the family into the decision-making process in order to optimize patient outcomes; therefore, this will hopefully also improve HCP-patient relationships. This article is based on findings from previous studies and a debate; it does not report any new studies with human participants or animals performed by any of the authors.

\section{BENEFITS OF FAMILY INVOLVEMENT IN CONSULTATIONS WITH HCPS}

Involving families in consultations can support patient engagement, HCP-patient understanding and personalized care in several ways. PwMS may find clinical encounters distressing; family members can provide emotional support and, in some situations, help PwMS to be calm, open and honest during the HCP consultation. This openness can, in turn, aid the convergence of the professional and patient perspectives that is essential to allow patient-centred care and shared decision-making [7].

As part of a patient-centred approach, family support may help the PwMS better recall and articulate any changes in symptoms. Some behavioural, affective, cognitive and neuropsychiatric symptoms associated with MS-such as emotional dysfunction, dysphoria, agitation, anxiety and depression-are inherently subjective [22-24, 26, 27]. By observing and interacting with PwMS on a daily basis, family members can offer additional information and insights into the patient's symptoms and experiences. Additionally, by being removed from the direct experience of MS, family members may be better placed to give an accurate overview of the status of the PwMS during gaps between appointments. Self-reported perceptions of long-term trends in patient well-being have been shown to be strongly distorted by present circumstances and are disproportionately affected by events in the recent past [28]. In these situations, the slightly more removed perspective of the family member may provide the HCP with a more accurate and balanced view of any improvement or decline of symptoms. Involving family members may also promote a more holistic discussion by prompting conversations that address the practical and social issues associated with the disease, such as employment decisions and home adjustments. In particular, PwMS may prefer to discuss pregnancy and family planning in the presence of a partner, as this in particular has a direct impact on both individuals.

Along with helping HCPs to understand the impact of subjective invisible symptoms, support from family members may also help to bridge the HCP-PwMS communication gaps resulting from emotional, cognitive or neuropsychiatric symptoms that may otherwise impair discussions. Even without these additional challenges, PwMS sometimes express concerns about being 'bombarded' with facts in appointments and experiencing information overload [29]. Having a family member attend the consultation means that the PwMS and their relatives have received the same information, which means they can support each other's understanding and recall MS procedures and treatments, while reducing discordance and misunderstanding. In some cases, a family member can act as a linguistic interpreter $[30,31]$.

Research carried out in comparator conditions provides additional supporting evidence for the benefits of family members attending appointments. A study of 439 patients with heart failure or diabetes attending 88 primary HCPs found that $78 \%$ of those who included family members in the consultation felt more motivated to follow their doctor's advice, $77 \%$ 
understood the advice better and 73\% found the consultation helped them explain the condition to family and friends [32].

The influence of family members on patient motivation and decision-making extends beyond consultations. While PwMS commonly quote their neurologist as their main source of trusted information about MS and its management [33], they spend limited time with their neurologist or multidisciplinary team. Neurologists increasingly emphasize that lifestyle changes are an important element in MS care; for example, smoking and alcohol abuse increase the risk of all-cause mortality among PwMS by 2.0- and 7.6-fold, respectively [34]. Similarly, evidence shows that each additional year of smoking after diagnosis accelerated the time to conversion to secondary progressive MS by $4.7 \%$ [35]. Families can help with early intervention and support PwMS to adhere to treatment and lifestyle changes, such as exercise, maintaining activities of daily living (including intellectual stimulation), smoking cessation, sensible drinking and changes to diet [36].

Families can also positively influence decision-making and MS management by helping PwMS to accept their diagnosis and overcome 'analysis paralysis' around making care decisions. 'Analysis paralysis' or overthinking results in delays to decisions or actions [37] and is particularly relevant in MS given the importance of starting treatment as early as possible [36]. Family members can offer PwMS a more objective view to support rational decisionmaking and reassure patients that they will provide practical support.

Treatment adherence is a major problem in MS, with estimates ranging from $88 \%$ down to only $28 \%$ [38]. Several factors may contribute to poor adherence, including long periods of remission, the inherent unpredictability of MS, inadequate knowledge of MS or its treatments, fear of needles, side effects, low self-efficacy, cognitive deficits and psychological problems. PwMS with a co-morbid mood or anxiety disorder are almost five times more likely to experience problems with adherence to DMTs [38]. Family members may be able to support the PwMS to overcome some of these issues or, at least, bring them to the attention of the healthcare team.

\section{ISSUES REGARDING FAMILY INVOLVEMENT IN CONSULTATIONS WITH HCPS}

As the previous section suggests, including family members in consultations and MS care can offer valuable insights and support for both the PwMS and the HCP, as well as facilitate patient-centred care and shared decisionmaking. While the debate highlighted these benefits, there was also recognition that challenges exist, which mean that family involvement cannot be considered a positive in all circumstances.

Depending on the healthcare system, involving family members in consultations may increase time pressure and make it harder for the HCP to cover important topics within the time available. Including another voice in the conversation is an additional pressure in itself, and it must be recognized that the informational and educational needs of family members may differ from those of PwMS. While these different needs may promote more holistic discussions about the care of the PwMS, they also introduce an additional potential for competing priorities for the HCP to navigate.

Depending on the specific relationship dynamic, the presence of family members in consultations may have an inhibitory effect on the willingness of the PwMS to be open and honest about certain sensitive aspects of MS that carry a societal stigma. For example, up to $75 \%$ of PwMS report experiencing sexual dysfunction [39]. In men with MS this may mean erectile or ejaculatory problems, and women with MS may develop vaginal dryness. Both sexes may experience reduced libido and difficulties reaching orgasm [39]. The presence of family members may discourage PwMS from discussing these problems as well as other potentially embarrassing, but common, problems, such as bladder and bowel dysfunction. An overall reluctance to discuss these issues may explain why, in general, PwMS report low levels of treatment satisfaction for these particular 
issues [40]. While the effect on sex life is often one of the most distressing aspects of a chronic disease, generally, many doctors are reluctant to initiate conversations because of concerns about causing offence or embarrassment. The presence of a third party in an appointment may easily be viewed as a further barrier to addressing these important issues.

Patients may wish to maintain a strong separation between their condition and their home life, and to minimize the exposure of family members to the burden of care. Having another person present in a consultation means that a patient cannot 'filter' the content of a shared consultation. In a study of patients with heart failure or diabetes, $12 \%$ of those whose consultation included family members or friends reported that the shared consultation created new disagreements [32]. Furthermore, 9\% and $4 \%$ felt that relatives and HCPs, respectively, disclosed more information than they should have done [32]. A shared consultation may also bring home the reality of MS to the family member. Issues such as the uncertainty of the MS trajectory, concerns over disability and the impact on family life can cause psychological problems for family members. Evidence from epilepsy shows a negative reciprocal relationship between disease-related depression and anxiety in family members, and increased psychological problems for the person with epilepsy [41]. There is no reason to suppose that the same reciprocal relationship would not apply to PwMS and their families, although further MS-specific studies are needed.

Changing relationship dynamics and external tensions may negatively affect communication within appointments. This is particularly important for paediatric PwMS, given the increasing recognition of paediatric-onset MS [19] and the well-documented changes in relationship between children and adults during puberty [42]. While they are younger, paediatric PwMS rely heavily on their parents for practical and emotional support for management of their MS. As the child matures, they become less dependent on their parents, and the psychosocial transition and change in roles experienced can cause familial stress [19, 43]. Adolescents PwMS may be frustrated by overbearing and hypervigilant parental behaviours at a time that teenagers want to assert their independence $[19,43]$. A desire for independence may reduce DMT adherence in adolescence and this may be exacerbated by teenagers' sense of omnipotence, cognitive limitations and inexperience of assessing longterm risks and benefits [19]. The model of constructive involvement of families in consultations and decision-making-without disrupting care, the transition between services or undermining the adolescent's autonomy-will need to change as the teenager matures or the family dynamics shift.

In some cases, a lack of family involvement in consultations and care may be a deliberate choice on the part of the PwMS. This may be a result of a desire to minimize the burden of care on the individuals around them. PwMS may also want to avoid involving family members in their care in order to minimize the impact of the disease on their personal relationships. An individual whose historical role has been that of the primary 'breadwinner' or the 'head of the household' may not want to be perceived as disabled and in need of care. Indeed, fundamental changes in their familial role can be a source of distress and tension for the PwMS and other family members. HCPs may need to help the PwMS and their family negotiate the changed dynamics and encourage a reluctant person to accept help while redefining a personally meaningful role.

These challenges with family dynamics can have negative impacts outside of appointments. As previously mentioned, contact between PwMS and their HCP is limited, meaning that the opinions of family members can have a strong influence on decision-making, both inside and outside the healthcare setting. If family members have strong opinions about the care available or provided, this can negatively influence the PwMS in their decision-making and MS management. This may be especially the case where the wishes and opinions of the family member conflict with those of the PwMS or HCP. The cultural, social and religious background of the PwMS should be considered by the HCP, as it may influence the decisionmaking process. For example, some close family 
units or those with a strong patriarchal or matriarchal 'pyramid structure' may not want the PwMS to make decisions independently, which is a particularly important consideration given the increased prevalence of MS in female versus male individuals, and that this ratio continues to increase over time [44]. Moreover, some cultural and religious beliefs may promote a fatalistic view of an illness [7] or advise against specific or any medical interventions. For example, some cultures traditionally advocate bedrest for ill people, yet physical activity has psychological, physical and, possibly, cognitive benefits for PwMS $[45,46]$. The tension between the different sources of information can leave the PwMS conflicted. Undoubtedly, further research is needed into the impact of cultural, societal and religious beliefs on all aspects of MS, particularly as prevalence increases both in female individuals [44] and in regions such as Asia [14, 15] and Latin America [16, 17].

Overbearing involvement by family members may prevent PwMS from taking responsibility for management of, or education about, their MS. A study of 66 adolescent PwMS suggested that parents overestimated adherence compared with the patient's self-report (14\% and $41 \%$, respectively, estimated that the PwMS missed more than 20\% of doses) [47]. It is important that PwMS, particularly when young, learn and develop both the skills and understanding required to facilitate positive selfmanagement and self-sufficiency. While family member support in most situations will come from a positive place, such individuals cannot always be present to support the PwMS and, therefore, a more 'hands-off' approach to involvement in care may sometimes be in the best interests of the PwMS.

\section{OPTIMIZING COMMUNICATION WITH, AND SUPPORT FOR, FAMILIES OF PWMS}

The discussions highlight the number of considerations that need to be made when optimizing family involvement in MS. HCPs need to be mindful of the influence that family opinions and dynamics can have, both positive and negative, both inside and outside the medical setting. As with many aspects of MS, it is important to recognize that there is no 'onesize-fits-all' solution and that every PwMS is unique and will have different needs. In this section, we discuss some of the potential solutions to these challenges that were raised by both the panel and the audience during the debate.

Within appointments, despite the potential benefits of family member presence, it is important to remember that, as with any healthcare scenario, the PwMS has a right to privacy and confidentiality. This privacy can be particularly important when it gives the PwMS the confidence to discuss difficult or sensitive topics. In cases where PwMS are comfortable and happy for family members to attend the appointment alongside them, there may still be benefits to allowing space and opportunity for these types of private discussions. One approach is for the HCP to see the PwMS on his or her own for half the consultation. Family members can be admitted for the other half. This approach may be particularly useful when interacting with adolescent PwMS, as it gives them space for independence without excluding the parents entirely.

MS services could consider whether their service optimally balances the needs of PwMS and their families. The family's need for information and support, for example, may be more appropriately addressed separately outside the appointment with the PwMS or through other channels (e.g. internet resources). Families may benefit from the opportunity to voice their concerns in a less time-restricted environment and without adding to the burden of the PwMS. Peer support groups and in-clinic training days, as well as access to MS nurses or social workers, may all meet the needs of family members without requiring direct involvement in consultations.

If providing separate services for family members, it is important that the MS team work together to ensure that they deliver consistent and culturally sensitive messages. Separate education of families and PwMS may disrupt shared understanding of MS and the risks and benefits of management strategies. Family 
members, especially if not present during consultations or education, may find information on the internet that conflicts with that from HCPs. One solution to this is for HCPs to 'signpost' PwMS and their families to patient organizations, charities and other sources of reliable, accurate and tailored information.

\section{DISCUSSION}

MS affects the entire family, and family can be both the most influential factor to empower the patient and the biggest barrier to achieving optimal patient outcomes. HCPs should aim to understand the dynamics between these support persons and PwMS, and individualize consultations and information to enable optimal patientcentred care and shared decision-making.

Fundamentally, family involvement in care, regardless of the form that it takes, should be for the benefit of, and in accordance with the preference of, the PwMS. Family attendance in appointments works best when it helps the PwMS to receive better care and improve outcomes outside of the medical setting. Support for the family, although important, may be more effectively and appropriately dealt with outside of the HCP consultation.

In certain cases, such as the care of paediatric PwMS, family member presence in appointments will be necessary. Approximately, 3-10\% of MS cases first emerge during childhood or adolescence [48]. Neurologists now recognize that paediatric MS was historically underdiagnosed and undertreated [49]. HCPs need to seek opportunities to engage with the family and provide other support networks to develop individualized management approaches for children and adolescent PwMS [19]. However, family dynamics will evolve; therefore, to optimize outcomes, HCPs need to continuously monitor the desire of the PwMS for family involvement and any evolving dynamics, to avoid exacerbating tensions and triggering counterproductive behaviour.

This debate revealed several areas where additional studies are warranted; for example, determining the importance and influence that families' perceptions of care might have on patients' satisfaction with care. Incorporating family-reported outcomes into patient-reported outcome models may be one solution to help HCPs to better understand the impact that MS and treatment have on patients' and families' quality of life and activities. Studies could also address the impact of MS and management strategies on romantic relationships and family dynamics, and how families can help PwMS who may not want to be perceived as disabled to live full, active and personally meaningful lives.

Studies need to better understand the dynamics between HCPs, PwMS and families. The role of caregivers needs to be considered when developing future studies and interventions, as they are often instrumental to adherence and successful symptom management in PwMS. There is mounting evidence of psychological distress in caregivers of PwMS, but there are few services or interventions to ease caregiver burden. Future work may consider focusing on developing an intervention to improve psychological well-being in PwMS and caregivers [3].

The prevalence and incidence of MS is increasing globally, including in parts of Eastern and Southern Europe [11-13], Asia [14, 15] and Latin America [16, 17]. This global reach means that advice and guidelines around standards of patient-centred care will increasingly need to account for a greater diversity of cultural backgrounds and family structures and dynamics than might previously have been the case. While these different family dynamics may provide their own challenges, they are not necessarily counterproductive and can, when aligned with the HCP, offer a valuable support for PwMS and reinforce professional advice. HCPs, however, need to understand the dynamics between non-medical influencers and PwMS, and individualize consultations and information accordingly.

\section{CONCLUSIONS}

When considering how best to integrate family members and caregivers into the management of MS, HCPs need to understand the individual 
needs of PwMS. The level and form of involvement should be determined ultimately by what will benefit the PwMS, and HCPs will need to consider the PwMS' personal circumstances and family situation. Providing personalized, culturally sensitive and patient-centred care will ensure the best relationship between the HCP and PwMS, and help them to optimize outcomes overall. HCPs should consider the timing, type and amount of information to provide for each PwMS and their family, as well as how best to collaborate with them. Ultimately, HCPs must listen to and respect the decisions of PwMS, and ensure that, whatever the needs of the PwMS, they feel supported and empowered to manage their condition.

\section{ACKNOWLEDGEMENTS}

The members of the MS in the 21st Century Steering Group are as follows: Michelle Allan, Raed Alroughani, Alexey Boyko, Birgit Bauer, Trishna Bharadia, Elisabeth Gulowsen Celius, Maria Paz Giambastiani, Gavin Giovannoni, Jeremy Hobart, Jürg Kesselring, Dawn Langdon, Sarah A Morrow, Jocelyne Nouvet-Gire, Elisabeth Kasilingam, Paola Kruger, Alice Laroni, Celia Oreja-Guevara, Maija Pontaga, Stanca Potra, Peter Rieckmann, Jane Shanahan, MarjaLiisa Sumelahti, Heidi Thompson, Pieter van Galen, Patrick Vermersch, Mitzi Williams and David Yeandle. We thank the European Charcot Foundation audience members for their contributions to this debate.

Funding. The MS in the 21st Century initiative is financially supported by Merck KGaA, Darmstadt, Germany who also funded the journal's Rapid Service Fee. Merck CrossRef Funder ID: https://doi.org/10.13039/ 100009945.

Medical Writing and Editorial Assistance. Administrative support and editorial input were provided by Cello Health Communications. Medical writing assistance was provided by Cello Health Communications (Mark Greener, Owen Webb and Cassie Mackenzie).
Administrative support and medical writing assistance were funded by Merck KGaA, Darmstadt, Germany.

Authorship. All named authors meet the International Committee of Medical Journal Editors (ICMJE) criteria for authorship for this article, take responsibility for the integrity of the work as a whole and have given their approval for this version to be published.

Authors' Contributions. All authors contributed to the concept and design of the debate, the development of the content for the meeting, draft preparation of the manuscript, review and editing of the manuscript and final manuscript development. Jürg Kesselring, Alexey Boyko, Alice Laroni, Trishna Bharadia and Pieter van Galen all participated in the panel presentation.

Disclosures. Jürg Kesselring has received honoraria from Merck KGaA for MS in the 21st Century activities. Alexey Boyko received honoraria as member of working groups and advisory boards, and participated in clinical trials supported by Biogen, Schering, Merck KGaA, Teva, Novartis, Sanofi-Genzyme, Roche, Actelion, Biocad and Generium. Alice Laroni has received personal compensation for public speaking and advisory boards from Novartis, Sanofi Genzyme, Biogen, Merck KGaA, Roche and Teva, and has received research grants from the Italian Ministry of Health, the Italian Ministry of University and The Italian MS Foundation. Trishna Bharadia has received honoraria from Merck KGaA for MS in the 21st Century activities, and has received consulting and speaking fees from Parexel, Fishawack, Talkhealth, Teva, Roche, Novartis, WPP Health, Envision Pharma and AbbVie. Pieter van Galen has received honoraria from Merck KGaA for MS in the 21st Century activities, and has received consulting and speaking fees from Novartis, Merck NV-SA, Celgene R\&D Sarl, F. Hoffman-La Roche, NV Roche SA, Mylan GMBH and Excemed. Nektaria Alexandri is an employee of Merck KGaA. 
Compliance with Ethics Guidelines. This article is based on findings from previous studies and a debate; it does not report any new studies with human participants or animals performed by any of the authors.

Data Availability. Data sharing is not applicable to this article as no datasets were generated or analysed during the current study.

Open Access. This article is licensed under a Creative Commons Attribution-NonCommercial 4.0 International License, which permits any non-commercial use, sharing, adaptation, distribution and reproduction in any medium or format, as long as you give appropriate credit to the original author(s) and the source, provide a link to the Creative Commons licence, and indicate if changes were made. The images or other third party material in this article are included in the article's Creative Commons licence, unless indicated otherwise in a credit line to the material. If material is not included in the article's Creative Commons licence and your intended use is not permitted by statutory regulation or exceeds the permitted use, you will need to obtain permission directly from the copyright holder. To view a copy of this licence, visit http://creativecommons.org/licenses/by$\mathrm{nc} / 4.0 /$.

\section{REFERENCES}

1. Scolding N, Barnes D, Cader S, et al. Association of British Neurologists: revised (2015) guidelines for prescribing disease-modifying treatments in multiple sclerosis. Pract Neurol. 2015;15:273-9.

2. Dennison L, McCloy Smith E, Bradbury K, Galea I. How do people with multiple sclerosis experience prognostic uncertainty and prognosis communication? A qualitative study. PLOS ONE. 2016;11: e0158982.

3. Kidd T, Carey N, Mold F, et al. A systematic review of the effectiveness of self-management interventions in people with multiple sclerosis at improving depression, anxiety and quality of life. PLoS ONE. 2017;12:e0185931.
4. Boström K, Nilsagård Y. A family matter-when a parent is diagnosed with multiple sclerosis. A qualitative study. J Clin Nurs. 2016;25:1053-61.

5. Parker G. Disability, caring and marriage: the experience of younger couples when a partner is disabled after marriage. Br J Soc Work. 1993;23: 565-80.

6. Colligan E, Metzler A, Tiryaki E. Shared decisionmaking in multiple sclerosis. Mult Scler. 2017;23: 185-90.

7. Russel A. The social basis of medicine. 1st ed. Chichester: Wiley-Blackwell; 2009.

8. Hickey JV. Good communication with healthcare providers helped patients with multiple sclerosis to cope and adapt. Evid Based Nurs. 2004;7:124.

9. Alroughani RA. Improving communication with multiple sclerosis patients. Neurosciences (Riyadh). 2015;20:95-7.

10. de Seze J, Borgel F, Brudon F. Patient perceptions of multiple sclerosis and its treatment. Patient Prefer Adherence. 2012;6:263-73.

11. Pugliatti M, Rosati G, Carton $H$, et al. The epidemiology of multiple sclerosis in Europe. Eur J Neurol. 2006;13:700-22.

12. Boyko A, Smirnova N, Petrov S, Gusev E. Epidemiology of MS in Russia, a historical review. Mult Scler Demyelinating Disord. 2016;1:13.

13. Boyko A, Melnikov M. Prevalence and incidence of multiple sclerosis in Russian Federation: 30 years of studies. Brain Sci. 2020;10:305.

14. Eskandarieh S, Heydarpour P, Minagar A, Pourmand S, Sahraian MA. Multiple sclerosis epidemiology in East Asia, South East Asia and South Asia: a systematic review. Neuroepidemiology. 2016;46: 209-21.

15. Viswanathan S, Wah LM. A nationwide epidemiological study on the prevalence of multiple sclerosis and neuromyelitis optica spectrum disorder with important multi-ethnic differences in Malaysia. Mult Scler. 2019;25:1452-61.

16. Cristiano E, Rojas JI. Multiple sclerosis epidemiology in Latin America: an updated survey. Mult Scler J Exp Transl Clin. 2017. https://doi.org/10.1177/ 2055217317715050.

17. Ribeiro TAGJ, Duarte AL, da Silva DJ, et al. Prevalence of multiple sclerosis in Goiânia, Goiás, Brazil. Arq Neuropsiquiatr. 2019;77:352-6. 
18. Dobson R, Dassan P, Roberts M, Giovannoni G, Nelson-Piercy C, Brex PA. UK consensus on pregnancy in multiple sclerosis: 'Association of British Neurologists' guidelines. Pract Neurol. 2019;19: 106-14.

19. Mah JK, Thannhauser JE. Management of multiple sclerosis in adolescents - current treatment options and related adherence issues. Adolescent Health Med Ther. 2010;1:31-43.

20. Members of the MS21 Steering Group, Rieckmann $\mathrm{P}$, Centonze $\mathrm{D}$, et al. Unmet needs, burden of treatment, and patient engagement in multiple sclerosis: a combined perspective from the MS in the 21st Century Steering Group. Mult Scler Relat Disord. 2018;19:153-60.

21. Tintoré $\mathrm{M}$, Alexander M, Costello $\mathrm{K}$, et al. The state of multiple sclerosis: current insight into the patient/health care provider relationship, treatment challenges, and satisfaction. Patient Prefer Adherence. 2016;11:33-45.

22. Sumowski JF, Benedict R, Enzinger C, et al. Cognition in multiple sclerosis: state of the field and priorities for the future. Neurology. 2018;90: 278-88.

23. Johnen A, Bürkner P-C, Landmeyer NC, et al. Can we predict cognitive decline after initial diagnosis of multiple sclerosis? Results from the German National early MS cohort (KKNMS). J Neurol. 2019;266:386-97.

24. Ekmekci O. Pediatric multiple sclerosis and cognition: a review of clinical, neuropsychologic, and neuroradiologic features. Behav Neurol. 2017: 1463570 .

25. MS in the 21st Century. 2021. https:// msinthe21stcentury.com/en. Accessed 20 Oct 2021.

26. Shail MS. Neuropsychiatry in demyelination disease: using depression as a prodrome for early diagnosis and treatment of multiple sclerosis. Cureus. 2017;9:e1813.

27. Politte LC, Huffman JC, Stern TA. Neuropsychiatric manifestations of multiple sclerosis. Primary Care Companion J Clin Psychiatry. 2008;10:318-24.

28. Stull DE, Leidy NK, Parasuraman B, Chassany O. Optimal recall periods for patient-reported outcomes: challenges and potential solutions. Curr Med Res Opin. 2009;25:929-42.

29. Helland CB, Holmøy T, Gulbrandsen P. Barriers and facilitators related to rehabilitation stays in multiple sclerosis: a qualitative study. Int J MS Care. 2015;17:122-9.
30. Omole FS, Sow CM, Fresh E, Babalola D, Strothers H 3rd. Interacting with patients' family members during the office visit. Am Fam Phys. 2011;84: 780-4.

31. Sharp RJ, Hobson J. Patient and physician views of accompanied consultations in occupational health. Occup Med (Lond). 2016;66:643-8.

32. Rosland A-M, Piette JD, Choi H, Heisler M. Family and friend participation in primary care visits of patients with diabetes or heart failure: patient and physician determinants and experiences. Med Care. 2011;49:37-45.

33. Marrie RA, Salter AR, Tyry T, Fox RJ, Cutter GR. Preferred sources of health information in persons with multiple sclerosis: degree of trust and information sought. J Med Internet Res. 2013;15: e67-e67.

34. Jick SS, Li L, Falcone GJ, Vassilev ZP, Wallander M-A. Epidemiology of multiple sclerosis: results from a large observational study in the UK. J Neurol. 2015;262:2033-41.

35. Ramanujam R, Hedström A-K, Manouchehrinia A, et al. Effect of smoking cessation on multiple sclerosis prognosis. JAMA Neurol. 2015;72:1117-23.

36. Giovannoni G, Butzkueven H, Dhib-Jalbut $\mathrm{S}$, et al. Brain health: time matters in multiple sclerosis. Mult Scler Relat Disord. 2016;9:S5-48.

37. Kurien R, Paila A, Nagendra A. Application of paralysis analysis syndrome in customer decision making. Procedia Econ Finance. 2014;11:323-34.

38. Corallo F, Bonanno L, Di Cara M, et al. Therapeutic adherence and coping strategies in patients with multiple sclerosis: an observational study. Medicine. 2019;98:e16532.

39. Guo Z-N, He S-Y, Zhang H-L, Wu J, Yang Y. Multiple sclerosis and sexual dysfunction. Asian J Androl. 2012;14:530-5.

40. Wang G, Marrie RA, Fox RJ, et al. Treatment satisfaction and bothersome bladder, bowel, sexual symptoms in multiple sclerosis. Mult Scler Relat Disord. 2018;20:16-21.

41. Etemadifar S, Heidari M, Jivad N, Masoudi R. Effects of family-centered empowerment intervention on stress, anxiety, and depression among family caregivers of patients with epilepsy. Epilepsy Behav. 2018;88:106-12.

42. Suleiman AB, Dahl R. Parent-child relationships in the puberty years: insights from developmental neuroscience. Fam Relat. 2019;68:279-87. 
43. Tuffrey C, Pearce A. Transition from paediatric to adult medical services for young people with chronic neurological problems. J Neurol Neurosurg Psychiatry. 2003;74:1011-3.

44. Harbo HF, Gold R, Tintoré M. Sex and gender issues in multiple sclerosis. Ther Adv Neurol Disord. 2013;6:237-48.

45. Morrison JD, Mayer L. Physical activity and cognitive function in adults with multiple sclerosis: an integrative review. Disabil Rehabil. 2017;39: 1909-20.

46. Fasczewski KS, Gill DL, Rothberger SM. Physical activity motivation and benefits in people with multiple sclerosis. Disabil Rehabil. 2018;40: 1517-23.

47. Schwartz CE, Grover SA, Powell VE, et al. Risk factors for non-adherence to disease-modifying therapy in pediatric multiple sclerosis. Mult Scler. 2018;24:175-85.

48. Ghezzi A, Baroncini D, Zaffaroni M, Comi G. Pediatric versus adult MS: similar or different? Mult Scler Demyelinating Disord. 2017;2:5.

49. Alroughani R, Boyko A. Pediatric multiple sclerosis: a review. BMC Neurol. 2018;18:27. 